\title{
Profiling the human response to physical exercise: a computational strategy for the identification and kinetic analysis of metabolic biomarkers
}

Michael Netzer ${ }^{1}$, Klaus M Weinberger ${ }^{1,3,4}$, Michael Handler ${ }^{1}$, Michael Seger ${ }^{1}$, Xiaocong Fang ${ }^{5}$, Karl G Kugler $^{2}$, Armin Graber $^{2}$ and Christian Baumgartner ${ }^{1^{*}}$

\begin{abstract}
Background: In metabolomics, biomarker discovery is a highly data driven process and requires sophisticated computational methods for the search and prioritization of novel and unforeseen biomarkers in data, typically gathered in preclinical or clinical studies. In particular, the discovery of biomarker candidates from longitudinal cohort studies is crucial for kinetic analysis to better understand complex metabolic processes in the organism during physical activity.

Findings: In this work we introduce a novel computational strategy that allows to identify and study kinetic changes of putative biomarkers using targeted MS/MS profiling data from time series cohort studies or other crossover designs. We propose a prioritization model with the objective of classifying biomarker candidates according to their discriminatory ability and couple this discovery step with a novel network-based approach to visualize, review and interpret key metabolites and their dynamic interactions within the network. The application of our method on longitudinal stress test data revealed a panel of metabolic signatures, i.e., lactate, alanine, glycine and the short-chain fatty acids C2 and C3 in trained and physically fit persons during bicycle exercise.

Conclusions: We propose a new computational method for the discovery of new signatures in dynamic metabolic profiling data which revealed known and unexpected candidate biomarkers in physical activity. Many of them could be verified and confirmed by literature. Our computational approach is freely available as $R$ package termed BiomarkeR under LGPL via CRAN http://cran.r-project.org/web/packages/BiomarkeR/.
\end{abstract}

\section{Introduction}

In metabolomics the bioinformatics-driven search for highly-discriminatory biomarker candidates has become a key task in the biomarker discovery process with the objective of introducing novel biomarkers aiding in diagnosis or therapeutic management [1-4].

A wide spectrum of feature selection methods including filter, wrapper or embedded algorithms is available for the identification of significant features in biomedical datasets [5-9]. In particular filter algorithms calculate a measure (score), allowing to rank and prioritize putative biomarker

\footnotetext{
* Correspondence: christian.baumgartner@umit.at

${ }^{1}$ Research Group for Clinical Bioinformatics, Institute of Electrical, Electronic and Bioengineering, UMIT, 6060 Hall in Tirol, Austria

Full list of author information is available at the end of the article
}

candidates according to their predictive value [8]. However, research is still needed to provide bioinformatics methods for the scientific community that address paired/ dependent test hypotheses or time series studies. In addition, the quantitative analysis of networks has increasingly become an important technique for the biological interpretation of changes in disease-associated metabolic pathways, allowing the study of interconnectivity, interaction or correlation among analytes. For this type of analysis, different types of topological graph descriptors (e.g., parametric or partition-based entropy measures) can be used to analyze such complex biological networks $[10,11]$.

In this short report we propose a new computational strategy that identifies metabolic biomarker candidates according to their discriminatory ability from dependent 
samples, and we review and interpret them using a network-based approach. For the biomarker identification and prioritization step we apply a recently published filter algorithm, named Biomarker Identifier (BI), which calculates a score measure for every analyte, representing the discriminatory ability in terms of the product of sensitivity and specificity, and in an analogous way for paired samples [12]. After BI prioritization we apply a new method to infer a network from the data by calculating analyte ratios, representing interactions of analyte pairs in the network. This discovery step aims at verifying metabolites selected from the first step, and reviewing identified highly discriminatory analyte pairs according to their connectivity strength within the network. This connectivity network permits scientists to review single and multiple pathway reactions, e.g., by mapping this information on biochemical network databases like KEGG [13] for identifying functional changes or abnormalities in human metabolism. Finally, we demonstrate results of this approach using targeted MS/ MS profiling data for the search of metabolic signatures in physically fit persons during bicycle exercise, yielding known and partly unexpected interactions among analytes of physical activity.

\section{Computational strategy}

\section{Step 1: Feature ranking and prioritization model}

We apply the so-called BI model for selecting and prioritizing analytes into classes of weak, moderate and strong predictors, addressing both dependent and independent test hypotheses. In this work we focus in particular on metabolites changing over time for a given cohort (paired or dependent sample). The paired BI $(\mathrm{pBI})$ is thus defined as [12]:

$$
\begin{aligned}
& p B I=\lambda \cdot D A^{*} \cdot \sqrt{\left|\frac{\Delta_{\text {change }} \mid}{C V}\right|} \cdot \operatorname{sign}\left(\Delta_{\text {change }}\right), \\
& \Delta_{\text {change }}= \begin{cases}\Delta & \text { if } \Delta \geq 1 \\
-\frac{1}{\Delta} \text { else }\end{cases}
\end{aligned}
$$

where $\lambda$ is a scaling factor, $D A^{*}$ is a discriminance measure defined as percent change of metabolite levels in one direction versus baseline and $\Delta_{\text {change }}$ represents the median percent change. $C V$ is the coefficient of variation and is set to 1 if $C V>1$ by default to consider solely data distributions with smaller variance [12].

\section{Step 2: Network inference}

By definition, a network $G$ is defined as a set of vertices $V$ which are connected by edges $E: G=(V, E)$ [14]. Inferring the network includes three steps: (i) calculating all ratios $R$ between metabolites $M$ which represent chemical interactions, where $r_{i j}=\left|\log _{2}\left(\frac{m_{i}}{m_{j}}\right)\right|$ with $i>j$, and $m \in M, r \in R$. The logarithm induces symmetry of the ratios and their reciprocals, respectively. Note that by definition the metabolite concentrations must be positive $(m \geq 0)$; (ii) computing pBI scores $s_{i j}, s \in S$ on the logarithmic ratios $R$ and (iii) constructing a graph $G$ with:

$$
G_{i j}=\left\{\begin{array}{l}
1 \text { if }\left|s_{i j}\right|>\tau \\
0 \text { else, }
\end{array}\right.
$$

for $i, j \in 1, \ldots,|M|$. A ratio $r \in R$ is designated as a putative pathway reaction of the form $\mathrm{A} \rightarrow \mathrm{B}$, where a reactant $A$ is metabolized into a product $B$ via single or multiple reaction paths. To consider significant predictor pairs in the network the threshold $\tau$ has been evaluated using controlled simulated data in form of $D(D \sim$ $N(10,1)$, see "Additional file 1 ") as proposed by Guo et al. [15]. Next, we inferred the network for different values for $\tau$ and used vertices (metabolites) with at least one edge (i.e., degree $>0$ ) as input for classification and calculated the mean accuracy of the classifier using 10fold cross-validation (see "Additional file 1").

In contrast to a static network, typically constructed from data of independent case/control studies, a kinetic network can be inferred on single analytes or analyte pairs (as done in this work) with changes in levels greater than the fixed threshold $\tau$ at timepoint $t_{x}$ vs. baseline $\left(t_{0}\right)$, representing the dynamics of circulating metabolites over time.

Coupling step 1 with step 2 of our discovery strategy allows for verifying preselected metabolites of step 1 as highly connected vertices (hubs) in the network in step 2. Note that a high degree of interconnectivity of a hub (i) represents analogously a high discriminatory and predictive value of this hub, and (ii) embedded in a network of pathway reactions a key role of normal or abnormal metabolism.

Our computational approach is freely available as $R$ package termed BiomarkeR under LGPL via CRAN http://cran.r-project.org/web/packages/BiomarkeR/. We chose to implement our method in $\mathrm{R}$ because of the broad abundance of this programming language in the bioinformatics community and its open source nature. Additionally, there is a multitude of packages available for the handling and analysis of network-based data (e.g., igraph[16], QuACN[11] or BioNet[17]).

\section{Bicycle stress test}

We here present a new computational method for the search for stress biomarkers in physically fit persons using targeted MS/MS profiling technology. Using this approach 60 analytes were identified by applying 
characteristic mass transitions in multiple reaction monitoring, precursor and neutral loss scans, and quantified using internal standards added to the samples [18].

Briefly, metabolite profiling of blood samples of a total of 30 active and physically fit individuals (22 males, 8 females) with a mean age of $38.33 \pm 7.16$ years and mean body mass index $23.88 \pm 2.50 \mathrm{~kg} / \mathrm{m}^{2}$ was carried out. The used data represents analyses of residual material of a standard sport-physical examination where each proband had to bicycle on an exercise bike for increasing steps of Watt (W) levels (each step $25 \mathrm{~W}$ ) until the individual's maximum capacity was reached.

Capillary blood samples were obtained from the ear lobe before starting the exercise (at rest, $t_{0}$ ), and at all Watt levels up to the individual's maximum performance $\left(t_{\max }\right)$. A total of 60 metabolites (lactate, amino acids and acyl carnitines) were measured in absolute concentration values $(\mu \mathrm{mol} / \mathrm{L})$ [19]. All individuals gave written informed consent to the attending physician.

\section{Results and discussion}

Using our computational approach the pBI priority model was applied as first step to preselect key metabolites from the measured pool of 60 analytes by computing scores at timepoint $t_{0}$ (at rest) vs. $t_{\max }$ (at individual maximum performance). Figure 1 shows the pBI scores, exhibiting five metabolites (i.e., lactate, alanine, glycine and the two shortchain acyl carnitines $\mathrm{C} 2$ and $\mathrm{C} 3$ ) categorized as strong predictors. Figure 2 demonstrates the corresponding dynamic network for $\tau=73$ again with lactate, alanine, C2 and C3

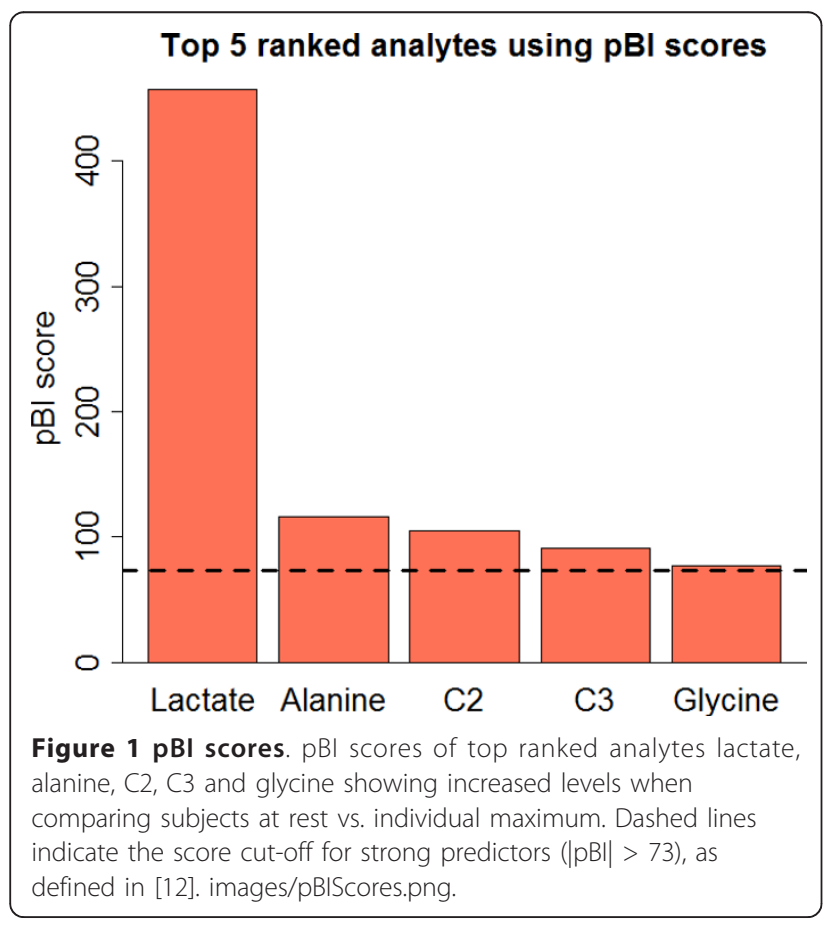

as major hubs, and now in the role as representatives of a panel of reaction pairs, coinciding with the univariate $\mathrm{pBI}$ metabolite ranking. Interestingly, using the controlled simulated data a threshold of $\tau=73$, which corresponds to the cut-off score for a strong predictor as defined in [12], led to the maximum mean accuracy using a K-nearestneighbor classifier [20], outperforming also commonly used correlation-networks [21] (see "Additional file 1").

To validate our findings a literature review and analysis of related KEGG pathways were performed. Among the total of 60 detected metabolites, our analysis revealed 5 key metabolites (lactate, alanine, glycine, and the two short-chain acyl carnitines C2 and C3) associated with physical exercise. These results are consistent with previous reports $[19,22,23]$. As is well known, anaerobic glycolysis is the main way for energy supply during exercise workout. Lactate is a major end product of the metabolism of glucose through the glycolytic pathway [23]. The skeletal muscle is the main organ producing large amounts of lactate. Typically, the production of lactate is greatly increased during exercise via the oxidative metabolism [23]. In our work, we detected a pBI score for lactate greater than 400 which represents a strong correlation with physical exercise. The known biochemical or physiologic effects of carnitine suggest that supplementation of carnitine may improve exercise performance $[24,25]$. First, carnitine is required for mitochondrial fatty acid oxidation, which would permit glucose utilization to decrease, and thus preserve muscle glycogen content and ensure maximal rates of oxidative ATP production $[24,26]$. Second, generation of acetylcarnitine would potentially decrease acetyl-CoA content, relieving inhibition of pyruvate dehydrogenase and decrease the production of lactate $[23,24,27]$. All of these can potentially improve physical performance during high-intensity exercise. Besides, recent studies demonstrated that short term administration of glycine propionyl-L-carnitine (GPLC) significantly elevates levels of nitric oxide metabolites at rest and in response to reactive hyperemia [28-30], and can also enhance exercise performance in healthy, trained individuals [28]. Carnosine is synthesized in skeletal muscle from L-histidine and A-alanine amino acids [22]. One important physiological role of carnosine is the maintenance of acid-base homeostasis [22,31]. Studies have shown that supplementation with A-alanine or exercise can increase muscle carnosine content and therefore total muscle buffer capacity with the potential to cause improvements in physical exercise $[19,22]$.

Overall, more than 20 pathways were revealed. We selected the most related pathways which include at least 2 metabolites identified by our coupled 2-step discovery step (Table 1). It shows that the citrate cycle (TCA cycle), multiple amino acid and fatty acid metabolisms are greatly activated in physical exercise. 


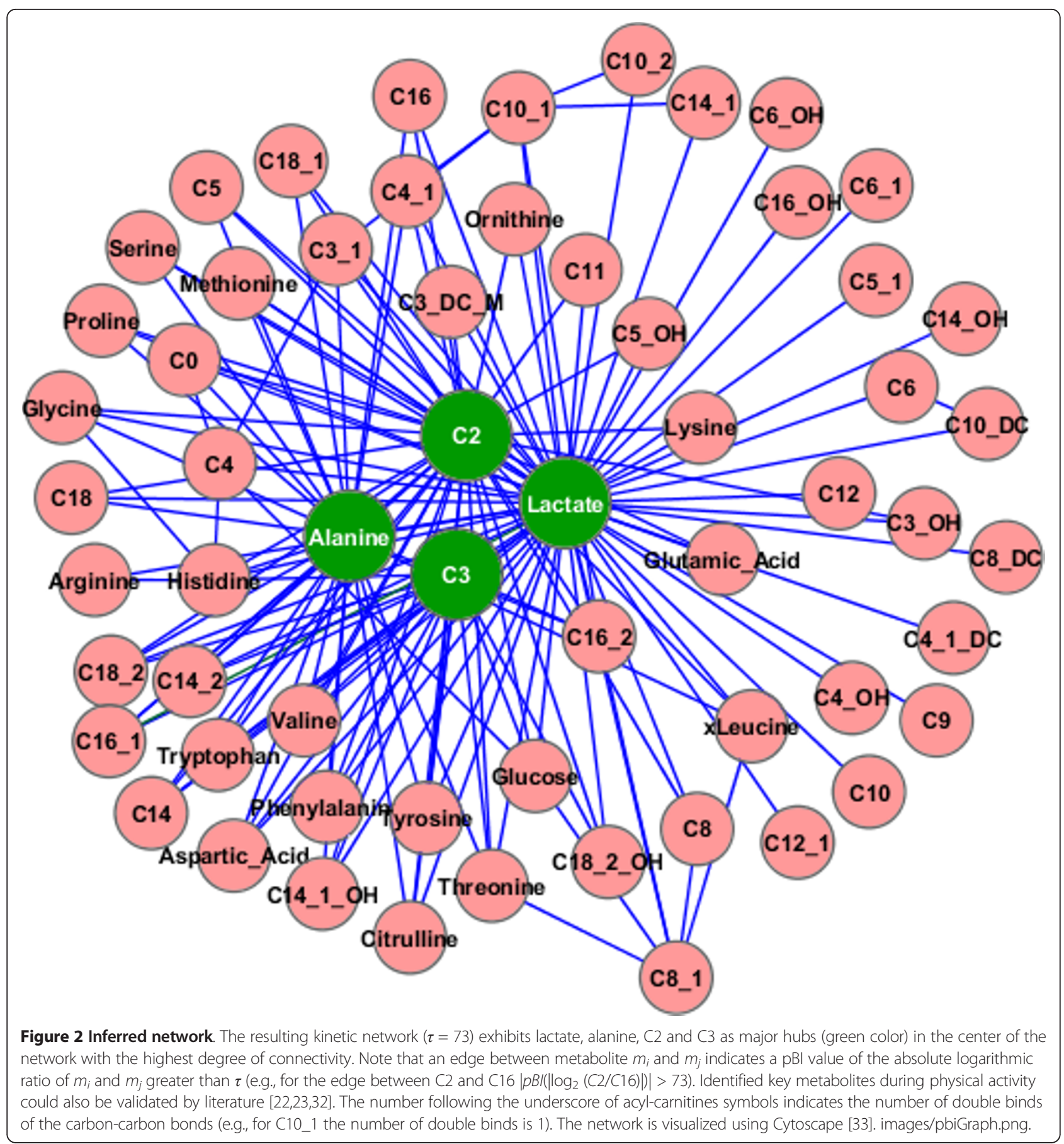

\section{Conclusion}

We have introduced a powerful tool for the search, prioritization and network analysis of putative biomarker candidates in metabolomic studies. Our 2-step approach has several benefits: 1) BI can be applied to dependent samples, calculating absolute scores for prioritizing biomarker candidates into classes of weak, moderate or strong predictors, and computes positive and negative scores, indicating whether the metabolites concentration is increased or decreased compared to its reference. 2) The proposed approach allows to review and interpret findings and thus aids in biochemical interpretation of (ab)normal metabolism by reviewing pathway reactions within the network.

Using our coupled 2-step discovery strategy, we were able to identify and confirm multiple metabolites, i.e., lactate, glycine, alanine, $\mathrm{C} 2$ and $\mathrm{C} 3$ that are closely associated with metabolism of physical activity $[22,23,32]$. 
Table 1 KEGG Pathways

\begin{tabular}{|c|c|c|}
\hline KEGG ID & KEGG pathway & Related metabolites \\
\hline Map00330 & Arginine and proline metabolism & $\begin{array}{l}\text { Alanine, aspartate, ornithine, proline, citrulline, arginine, glutamic acid, lysine, methionine, } \\
\text { glycine }\end{array}$ \\
\hline Map00020* & Citrate cycle & Glycose, leucine, valine, arginine, proline, tyrosine, alanine, aspartate, glutamic acid \\
\hline Map00260 & Glycine, serine and threonine metabolism & Serine, glycine, threonine, valine, aspartate, lysine, proline, methionine, leucine, arginine \\
\hline Map00250 & $\begin{array}{l}\text { Alanine, aspartate and glutamate } \\
\text { metabolism }\end{array}$ & aspartate, arginine, histidine, glutamic acid, alanine, proline, lysine, serine, glycine, glucose \\
\hline Map00620* & Pyruvate metabolism & Glycine, lactate, serine, leucine, lysine, valine, threonine \\
\hline Map00640 & Propanoate metabolism & Alanine, lactate, leucine, methionine, valine \\
\hline Map00270 & Cysteine and methionine & Methionine, threonine, alanine, aspartic acid, glycine, serine \\
\hline Map00473 & D-alanine metabolism & Alanine, aspartate, glutamate, glucose, ornithine \\
\hline Map00410 & $\beta$-alanine metabolism & Aspartate, alanine, histidine, lysine, arginine \\
\hline Map00460 & Cyano amino acid metabolism & Serine, glycine, alanine, glutamate, aspartate \\
\hline Map00300 & Lysine biosynthesis & Glycine, serine, threonine, lysine, alanine \\
\hline Map00710 & $\begin{array}{l}\text { Carbon fixation in photosynthetic } \\
\text { organisms }\end{array}$ & Tyrosine, phenylalanine, alanine, aspartate, glycose \\
\hline Map00071* & Fatty acid metabolism & Multiple carnitines, alanine, aspartate \\
\hline
\end{tabular}

Identified KEGG pathways in which metabolites selected by the inferred network are shown. The asterisks $\left({ }^{*}\right)$ indicate metabolic pathways associated with physical exercise (see also section "Results and discussion").

\section{Additional material}

Additional file 1: Simulation results. This PDF file contains an additional figure comparing mean accuracies for different thresholds using controlled simulated data and a K-nearest-neighbor as classifier.

\section{Acknowledgements}

This work was supported by the Austrian Genome Research Program GENAU (Bioinformatics Integration Network, BIN III). The authors also thank Matthias Dehmer and Laurin Müller for helpful discussion.

\section{Author details}

${ }^{1}$ Research Group for Clinical Bioinformatics, Institute of Electrical, Electronic and Bioengineering, UMIT, $6060 \mathrm{Hall}$ in Tirol, Austria. ${ }^{2}$ Institute for Bioinformatics and Translational Research, UMIT, $6060 \mathrm{Hall}$ in Tirol, Austria. ${ }^{3}$ Biocrates Life Sciences AG, Innrain 66/2, 6020 Innsbruck, Austria. ${ }^{4}$ Bavarian Nordic GmbH, Fraunhoferstr. 13, 82152 Martinsried, Munich, Germany. ${ }^{5}$ Zhongshan Hospital, Fudan University, 200032 Xuhui District, Shanghai, China.

\section{Authors' contributions}

MN and CB designed and wrote the manuscript. KW and AG performed mass spectrometry analysis and joined in data interpretation. KK and MN drafted and developed the network inference method. XF performed pathway analysis and interpretation. MN, MH, MS and KK designed and implemented the BiomarkeR package. All authors have read and approved the final manuscript.

\section{Competing interests}

The authors declare that they have no competing interests.

Received: 3 August 2011 Accepted: 19 December 2011 Published: 19 December 2011

\section{References}

1. Lewis GD, Asnani A, Gerszten RE: Application of metabolomics to cardiovascular biomarker and pathway discovery. J Am Coll Cardiol 2008, 52(2):117-123.

2. Boccard J, Veuthey JL, Rudaz S: Knowledge discovery in metabolomics: an overview of MS data handling. J Sep Sci 2010, 33(3):290-304.
3. Baumgartner C, Osl M, Netzer M, Baumgartner D: Bioinformatic-driven search for metabolic biomarkers in disease. J Clin Bioinforma 2011, 1:2.

4. Mamas M, Dunn WB, Neyses L, Goodacre R: The role of metabolites and metabolomics in clinically applicable biomarkers of disease. Arch Toxicol 2011, 85:5-17.

5. Hall MA, Holmes G: Benchmarking Attribute Selection Techniques for Discrete Class Data Mining. IEEE T Knowl Data Eng 2003, 15:1437-1447.

6. Enot DP, Beckmann M, Overy D, Draper J: Predicting interpretability of metabolome models based on behavior, putative identity, and biological relevance of explanatory signals. Proc Natl Acad Sci USA 2006, 103(40):14865-14870.

7. Saeys $Y$, Inza I, Larrañaga P: A review of feature selection techniques in bioinformatics. Bioinformatics 2007, 23:2507-2517.

8. Netzer M, Millonig G, Osl M, Pfeifer B, Praun S, Villinger J, Vogel W, Baumgartner C: A new ensemble-based algorithm for identifying breath gas marker candidates in liver disease using ion molecule reaction mass spectrometry. Bioinformatics 2009, 25(7):941-947.

9. Inza I, Calvo B, Armañanzas R, Bengoetxea E, Larrañaga P, Lozano JA: Machine learning: an indispensable tool in bioinformatics. Methods $\mathrm{Mol}$ Biol 2010, 593:25-48

10. Dehmer M, Barbarini N, Varmuza K, Graber A: Novel topological descriptors for analyzing biological networks. BMC Struct Biol 2010, 10:18.

11. Mueller LAJ, Kugler KG, Dander A, Graber A, Dehmer M: QuACN: an R package for analyzing complex biological networks quantitatively. Bioinformatics 2011, 27:140-141.

12. Baumgartner $C$, Lewis GD, Netzer M, Pfeifer B, Gerszten RE: A new data mining approach for profiling and categorizing kinetic patterns of metabolic biomarkers after myocardial injury. Bioinformatics 2010, 26(14):1745-1751.

13. Kanehisa M, Goto S: KEGG: kyoto encyclopedia of genes and genomes. Nucleic Acids Res 2000, 28:27-30.

14. Tang Y, Dogru AH, Kurfess FJ, Tanik MM: Computing Cyclomatic Complexity with Cubic Flowgraphs. Journal of Systems Integration 2001, 10:395-409.

15. Guo Y, Graber A, McBurney RN, Balasubramanian R: Sample size and statistical power considerations in high-dimensionality data settings: a comparative study of classification algorithms. BMC Bioinformatics 2010, 11:447.

16. Csardi G, Nepusz T: The igraph software package for complex network research. InterJournal 2006 [http://igraph.sf.net], Complex Systems:1695.

17. Beisser D, Klau G, Dandekar T, Mueller T, Dittrich M: BioNet an R-package for the Functional Analysis of Biological Networks. Bioinformatics 2009, 26:1129-1130. 
18. Baumgartner C, Graber A: In Successes and new directions in data mining. Volume 7. Idea Group Inc; 2007:141-166, Data mining and knowledge discovery in metabolomics.

19. Weinberger KM: Metabolomics in diagnosing metabolic diseases. Ther Umsch 2008, 65(9):487-491.

20. Parry RM, Jones W, Stokes TH, Phan JH, Moffitt RA, Fang H, Shi L, Oberthuer A, Fischer M, Tong W, Wang MD: k-Nearest neighbor models for microarray gene expression analysis and clinical outcome prediction. Pharmacogenomics J 2010, 10(4):292-309.

21. Mutwil M, Usadel B, Schütte M, Loraine A, Ebenhöh O, Persson S: Assembly of an interactive correlation network for the Arabidopsis genome using a novel heuristic clustering algorithm. Plant Physiol 2010, 152:29-43.

22. Artioli GG, Gualano B, Smith A, Stout J, Lancha AH: Role of beta-alanine supplementation on muscle carnosine and exercise performance. Med Sci Sports Exerc 2010, 42(6):1162-1173.

23. van Hall G: Lactate kinetics in human tissues at rest and during exercise. Acta Physiol (Oxf) 2010, 199(4):499-508.

24. Brass EP, Hiatt WR: The role of carnitine and carnitine supplementation during exercise in man and in individuals with special needs. J Am Coll Nutr 1998, 17(3):207-215.

25. Ahmaidi S, Granier P, Taoutaou Z, Mercier J, Dubouchaud H, Prefaut C: Effects of active recovery on plasma lactate and anaerobic power following repeated intensive exercise. Med Sci Sports Exerc 1996, 28(4):450-456.

26. Costill DL, Coyle E, Dalsky G, Evans W, Fink W, Hoopes D: Effects of elevated plasma FFA and insulin on muscle glycogen usage during exercise. J Appl Physiol 1977, 43(4):695-699.

27. Bremer J: Pyruvate dehydrogenase, substrate specificity and product inhibition. Eur J Biochem 1969, 8(4):535-540.

28. Jacobs PL, Goldstein ER, Blackburn W, Orem I, Hughes JJ: Glycine propionyl-L-carnitine produces enhanced anaerobic work capacity with reduced lactate accumulation in resistance trained males. J Int Soc Sports Nutr 2009, 6:9.

29. Smith WA, Fry AC, Tschume LC, Bloomer RJ: Effect of glycine propionyl-Lcarnitine on aerobic and anaerobic exercise performance. Int I Sport Nutr Exerc Metab 2008, 18:19-36.

30. Bloomer RJ, Tschume LC, Smith WA: Glycine propionyl-L-carnitine modulates lipid peroxidation and nitric oxide in human subjects. Int $J$ Vitam Nutr Res 2009, 79(3):131-141.

31. Harris RC, Tallon MJ, Dunnett M, Boobis L, Coakley J, Kim HJ, Fallow field JL, Hill CA, Sale C, Wise JA: The absorption of orally supplied beta-alanine and its effect on muscle carnosine synthesis in human vastus lateralis. Amino Acids 2006, 30(3):279-289.

32. Negrao CE, Ji LL, Schauer JE, Nagle FJ, Lardy HA: Carnitine supplementation and depletion: tissue carnitines and enzymes in fatty acid oxidation. J Appl Physiol 1987, 63:315-321

33. Cline MS, Smoot M, Cerami E, Kuchinsky A, Landys N, Workman C, Christmas R, Avila-Campilo I, Creech M, Gross B, Hanspers K, Isserlin R, Kelley R, Killcoyne S, Lotia S, Maere S, Morris J, Ono K, Pavlovic V, Pico AR, Vailaya A, Wang PL, Adler A, Conklin BR, Hood L, Kuiper M, Sander C, Schmulevich I, Schwikowski B, Warner GJ, Ideker T, Bader GD: Integration of biological networks and gene expression data using Cytoscape. Nat Protoc 2007, 2(10):2366-2382.

doi:10.1186/2043-9113-1-34

Cite this article as: Netzer et al:: Profiling the human response to physical exercise: a computational strategy for the identification and kinetic analysis of metabolic biomarkers. Journal of Clinical Bioinformatics 2011 1:34.

\section{Submit your next manuscript to BioMed Central and take full advantage of:}

- Convenient online submission

- Thorough peer review

- No space constraints or color figure charges

- Immediate publication on acceptance

- Inclusion in PubMed, CAS, Scopus and Google Scholar

- Research which is freely available for redistribution

Submit your manuscript at www.biomedcentral.com/submit
Biomed Central 